\title{
PURPURA FULMINANS IN A NEWBORN BABY
}

\author{
BY \\ R. L. VAN DER HORST \\ From the Department of Paediatrics, King Edward VIII Hospital, Durban, South Africa
}

(RECEIVED FOR PUBLICATION FEBRUARY 15, 1962)

The syndrome of purpura fulminans is a very rare disorder and, as far as can be ascertained, has never been reported in very young infants. The purpose of this paper is to record its occurrence in a newborn infant, to illustrate its severity and to draw attention to some unusual features.

\section{Case Report}

S.M., an Indian male infant, was the third child born normally at home on February 15, 1961. He was born with a number of raised red patches on the lateral aspects of both arms, right thigh and left cheek. These lesions slowly increased in size and after 24 hours were observed to be blueish in colour. Over a period of three days these lesions further increased in size, blistered in the centre, and the colour changed to a very dark blue. Meanwhile, fresh patches appeared on the left thigh, the lower back, buttocks and right cheek. At this stage, the infant was admitted to hospital because the mother was aware of the gravity of the child's condition.

The mother had attended an antenatal clinic frequently during her pregnancy and had at no time shown evidence of purpura. She denied taking any drugs, except iron and calcium tablets, and had never had transfusions of blood or blood products. The infant was given vitamin $\mathrm{K}$ by injection shortly after birth.

Family History. The mother, aged 24 years, and the father, aged 33 years, are of pure Hindu extraction and in good health. Their first child, a male, was born in June 1957 and had a similar condition to that of S.M. This infant had haematomata and bruising of the buttocks and legs as well as anaemia and thrombocytopenia. In spite of a blood transfusion, vitamin $\mathrm{K}$ and chlortetracycline therapy, he died on the ninth day of life. The cause of death was certified as haemorrhagic disease of the newborn. No autopsy was held.

A second child, a female, was born in June 1959, and had a blue patch on the right shoulder at birth. This disappeared spontaneously at 3 weeks of age, since when she has been in good health.

There is no history of bleeding or blood dyscrasia in other members of the family.

Physical Examination. The infant was normally developed and weighed $5 \mathrm{lb}$. $6 \mathrm{oz}$. He looked ill but was not distressed or febrile. The mucous membranes were pale and the sclerae were not icteric. There was slight pitting of the feet and legs and the cord was normal. Systematic examination did not reveal any abnormality. The confluent and indurated purple areas of the buttocks, thighs, left cheek and lateral aspects of both arms, had sharply defined edges. A black haemorrhagic patch was present on the lateral aspect of the right thigh. Some areas were discrete, while those that were purple in colour were surrounded by a red rim of inflammation about half an inch wide.

Clinical Course. The lesions merged into one another as they extended during the next three days, and bullae appeared. The colour changed from blue to dark blue to black. The skin became wrinkled, thickened, hard and necrotic, particularly over the buttocks (Fig. 1). Patches of epidermis were shed over these haemorrhagic sites. On the fourth day of life, the left lower limb was cold and indurated. It was purple in colour with a demarcation line above the ankle. No peripheral pulses were palpable although there was no apparent circulatory insufficiency since the left foet remained pink with normal capillary filling.

The lesions continued to spread in spite of further vitamin $\mathrm{K}$ therapy. On the sixth day of life, an infusion consisting of $1 \mathrm{~g}$. Of fibrinogen (Parenogen, Cutter) followed by $75 \mathrm{ml}$. of fresh whole blood were given. In spite of these procedures, the baby deteriorated still further (Figs. 2 and 3 ). There was a little bleeding from one nostril and the gums, producing blood-stained saliva. The stools became tarry black in colour, but there was no vomiting of fresh or altered blood. Increasing distension of the abdomen on the seventh day was followed by the infant's death early next morning.

Laboratory Data. The various blood findings are shown in Table 1. On the fifth day the haemoglobin was $7 \cdot 7 \mathrm{~g} . / 100 \mathrm{ml} .(52 \%)$ the platelet count $65,000 / \mathrm{c} . \mathrm{mm}$. and the fibrinogen level $60 \mathrm{mg} . / 100 \mathrm{ml}$. Before transfusion on the sixth day, the haemoglobin had dropped to $5.6 \mathrm{~g} . / 100 \mathrm{ml}$. (38\%), the platelets to 33,000 per c. $\mathrm{mm}$. and the fibrinogen level to $21.4 \mathrm{mg} . / 100 \mathrm{ml}$. In vitro clotting occurred in a non-siliconed glass test-tube at six minutes. Clot retraction was abnormal. There was considerable fall-out of red cells as described by Poe (1959). After the test-tube had been 'incubated' in the jacket pocket of the author, there was a very small clot of reduced volume and size. After 24 hours there was no dissolution of the clot which was still present and apparently of the same size. 


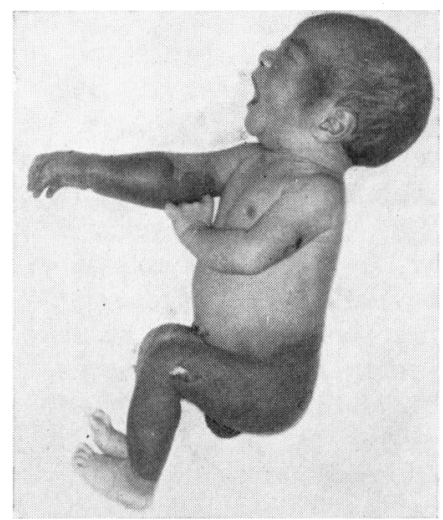

FIG. 1.-Side view taken on the fifth day of life showing, in part, the extent of the skin lesions.

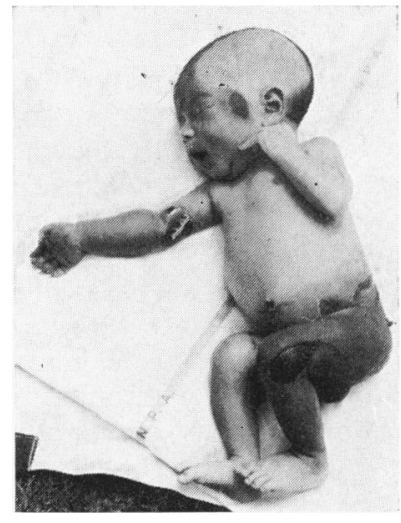

FIG. 2.-Left side view taken on the seventh day of life.

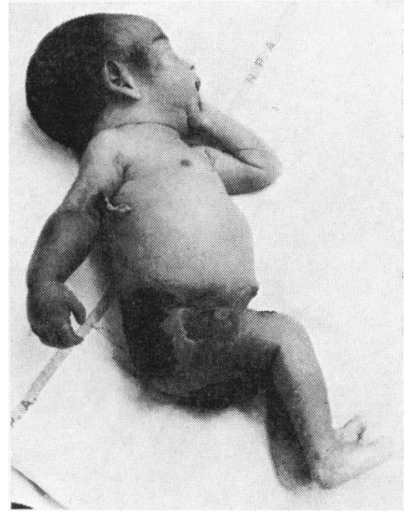

FIG. 3.-Right side view taken on the seventh day of life.
TABLE 1

VARIOUS BLOOD FINDINGS ON CASE DESCRIBED

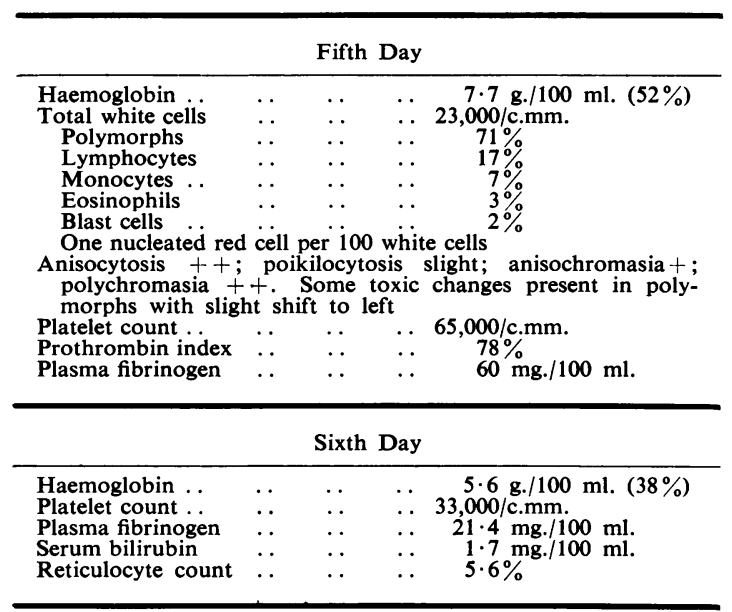

Autopsy. This revealed that the discoloration of the skin was caused by extensive haemorrhage chiefly into the subcutaneous fat. The main arteries and veins to the limbs did not contain any thrombi. The viscera were pale. The appendix, the descending colon, pelvic colon and rectum showed haemorrhagic infarction with sharp demarcation at the splenic flexure. The inferior mesenteric vein was thrombosed. The bladder was thickened and haemorrhagic, and this had led to ureteric obstruction and early bilateral hydronephrosis. There was no haemorrhage into the lungs, liver, kidneys, spleen or brain. All venous sinuses in the cranium were thrombosed. Microscopically there was extensive haemorrhage into the subcutaneous fat. In the older lesions haemorrhage was present with necrosis of the skin and secondary infection. The underlying muscle was scarcely involved. The widespread thrombosis was almost entirely limited

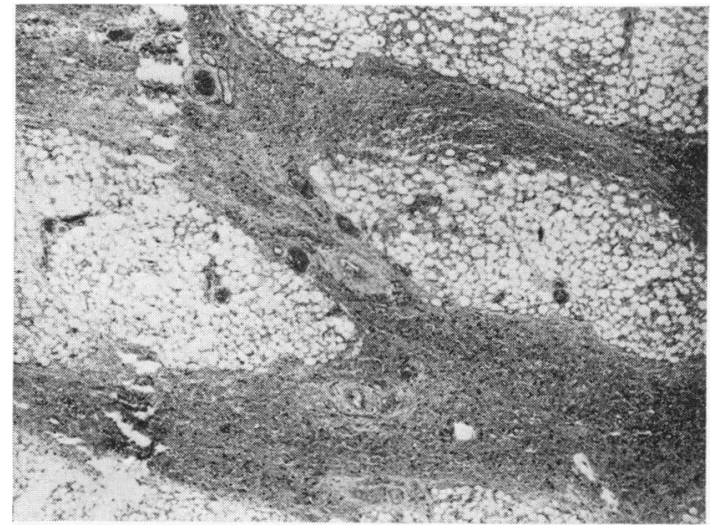

FIG. 4.--Subcutaneous fat showing extensive haemorrhage with thrombosed veins.

to small veins (Fig. 4) without necrosis or degeneration of the vessel walls. The lesions in the colon showed thrombosis of vessels and extravascular haemorrhage with extensive necrosis of the mucous membrane (Fig. 5). Vessels in the perinephric fat showed thrombosis with extravascular haemorrhage (Figs. 6 and 7). In addition to recent thrombosed veins, capillaries and arterioles, several thrombi were completely organized. No significant microscopic changes were seen in the parenchymatous organs.

\section{Discussion}

Intravascular Thrombosis and Purpura Fulminans. A number of reports have appeared under the various headings of purpura fulminans (Little, 1959; Crawford and Riddler, 1959; Heal and Kent, 1953; Chambers, Holyoke and Wilson, 1952), fulminating purpura (Turin, Mandel and Hornstein, 1959), purpura necrotica (Sheldon, 1947), purpura haemorrhagica (Hunt, 1938), gangrenous purpura (Dunn, 


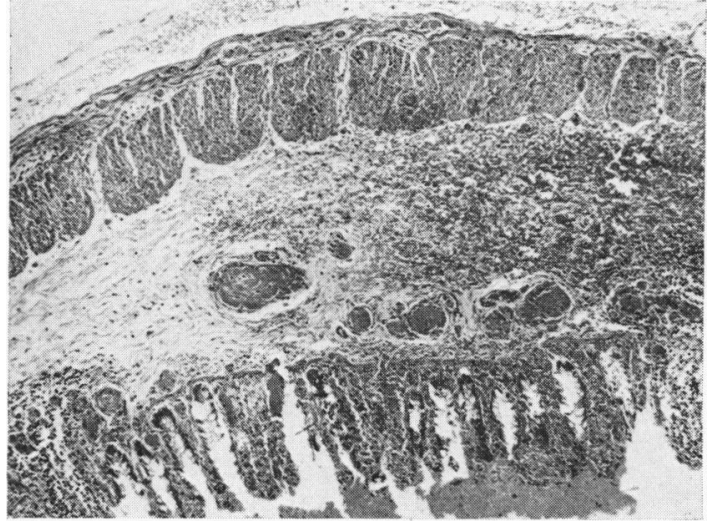

FIG. 5.-Intestine showing haemorrhage and thrombosed venules.

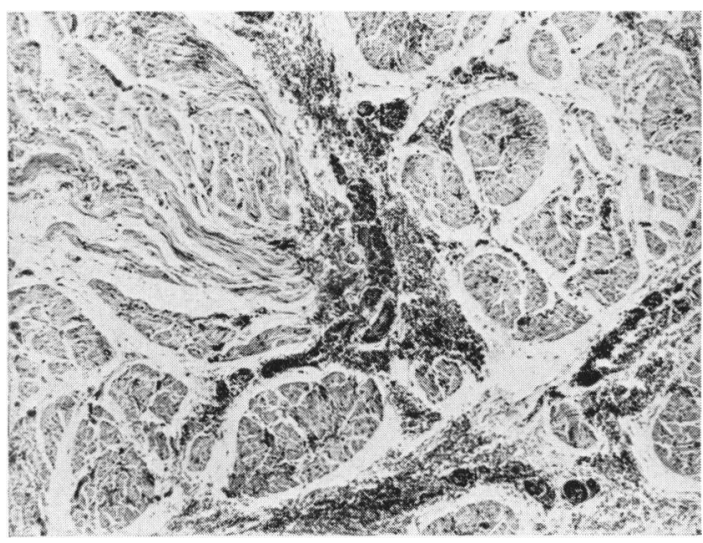

FIG. 6.-Bladder showing haemorrhage into bladder wall.

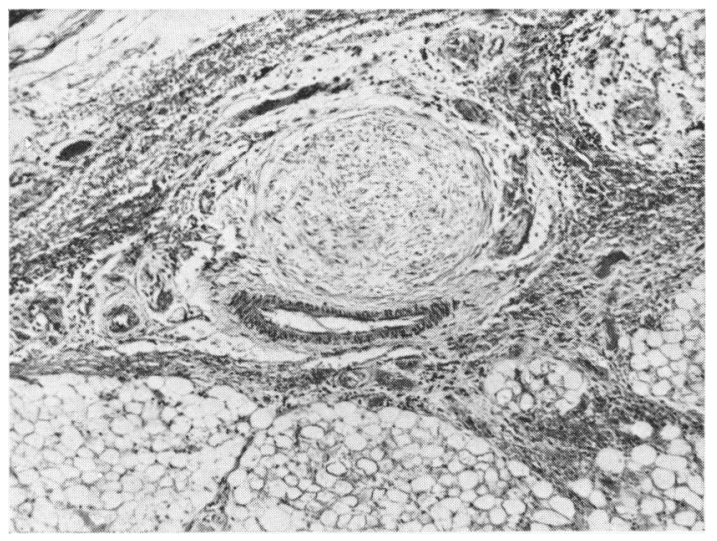

FIG. 7.-Renal capsule, showing organized thrombosed vein, recently thrombosed venules and widespread haemorrhage.
1951) and purpura gangrenosa (Taylor and Wright, 1956) in which the authors describe a very similar picture to the case under review. A well-defined clinical syndrome exists which has been referred to by the above titles. Those cases described more recently, including those of Seinfeld, Henniger and Hellman (1960), Marthaler, Pickel and Bütler (1957) and Koller, Gasser, Krüsi and de Muralt (1950) all seem to describe one and the same entity.

Most recent reports emphasize that the characteristic lesion in purpura fulminans is intravascular thrombosis. Extravasation of red blood cells occurs after necrosis of the vessel wall. Haemorrhage and depletion of coagulation factors are secondary phenomena. The somatic lesions of the disease are not typical 'purpura', but extensive gangrene.

Chambers et al. (1952) report a case of purpura fulminans with thrombosis of the inferior vena cava, common iliac veins, and branches of the pulmonary veins. McConnell and Weaver (1922) found extensive thrombosis in vessels of the bladder and intestine together with haemorrhage. This widespread internal damage has been infrequently recorded.

Accepting that purpura fulminans is essentially a thrombotic disease, then one can easily explain the severe features presented in this infant, namely, mesenteric vein thrombosis, haemorrhagic infarction of the intestine, which produced sequelae of obstruction, and similar damage to the bladder wall leading to ureteric obstruction and bilateral hydronephrosis.

Purpura fulminans appears to be the present accepted term for the syndrome. The disease is acute, rapidly progressive and frequently fatal because of the sudden and catastrophic occurrence of massive gangrene. The secondary haemorrhage becomes evident as extensive confluent ecchymoses which also in turn become gangrenous. The extremities are the usual sites affected, although the lesions may occur elsewhere. This condition is found more often in children than in adults. It may appear abruptly during convalescence from a relatively benign infectious disease such as rubella, rubeola, varicella, scarlet fever and streptococcal throat infection.

The significant features of purpura fulminans as illustrated by this case may be summarized as:

(a) Thrombosis of veins, capillaries and arterioles with extravasation of blood into surrounding tissues.

(b) Extensive ecchymoses which spread and become confluent.

(c) Hard necrotic areas of the skin with an inflammatory rim surrounding them. 
(d) A sharp definition of the surrounding skin from the lesions.

(e) Lack of absorption with the haemorrhage into the tissues progressing to gangrene.

The purpura, the low platelet counts and the poor clot formation suggested a coagulation disorder which prompted further investigation, particularly of the fibrinogen levels.

Coagulation Deficiencies in Purpura Fulminans. A number of cases of purpura fulminans have been reported in which there has been a deficit of factors concerned in coagulation. Little (1959), Marthaler et al. (1957), Heal and Kent (1953), Dyggve (1947), Knauer (1927) and Brühl (1931) describe cases with fibrinogen deficiency. Koller et al. (1950) reported on two cases of factor $\mathrm{V}$ deficiency. The case of Marthaler et al. had fibrinogen deficiency, factor $\mathrm{V}$ deficiency, thrombocytopenia and prothrombin deficiency. Webb, Dubbs and Conrad (1947) and Chambers et al. (1952) have described cases with coagulation defects without specific identification of the factors. A number of reports have called attention to variable degrees of thrombocytopenia (Little, 1959; Heal and Kent, 1953; Marthaler et al., 1957; Dick, Miller and Edmondson, 1934; Stoesser and Lockwood, 1938).

In the absence of external blood loss in this patient, the rapid development of a profound anaemia led to the conclusion that massive haemorrhage into the tissues was its most probable cause. There was no evidence of bleeding into the serous cavities nor of any haemolytic process since the infant was not clinically jaundiced. The raised reticulocyte count was in keeping with a bone marrow which was responsive to blood loss. Although it might have been expected, there was no hyperbilirubinaemia associated with 'enclosed haemorrhage', which has been reported in similar types of cases by Rausen and Diamond (1961).

The Fibrinogen Level. Quantitative estimation confirmed the low fibrinogen level which had already been suggested by the poor formation and retraction of the blood clot. Most investigators have found slight decrease in the fibrinogen concentration during the first days of life, but this is probably not sufficient to interfere with the formation of fibrin (van Creveld, 1959). Hypofibrinogenaemia and afibrinogenaemia do occur as congenital abnormalities of the clotting mechanism but their recognition is difficult, particularly in the newborn period. In view of the positive family history in this particular case, one had to consider the possibility of a genetically determined hereditary disorder. The mode of inheritance of congenital fibrinogen deficiency is not clear, but there is evidence that it is more adequately explained by the concept of partial or incomplete dominance (Schulman and Smith, 1957) than the classical theory of dominant and recessive genes.

A family suspected of congenital fibrinogen deficiency may be assessed by similar criteria of Glueck and Sutherland (1961). The plasma fibrinogen is estimated by Stirland's method (1956) in this hospital. The mean fibrinogen level is $300 \mathrm{mg}$./ $100 \mathrm{ml}$. with a normal range of $200 \mathrm{mg}$. $/ 100 \mathrm{ml}$. to $400 \mathrm{mg} . / 100 \mathrm{ml}$. Homozygous normal subjects have a level of $60 \%$ or more of the mean. Heterozygous normal subjects have a level of less than $40 \%$ of the mean, while homozygous abnormal subjects have less than $20 \%$ of the mean plasma fibrinogen level. The father, mother and sibling in the patient's family have fibrinogen levels under $40 \%$ of the mean (Table 2). They are, therefore, heterozygous normal subjects. Thus it is clear that the infant's family has a congenital deficiency of fibrinogen.

The Platelet Level. The thrombocytopenia of 65,000 and 33,000 per c.mm. was a striking feature of the disease in this infant. In an excellent review of congenital and neonatal thrombocytopenia, Kaplan (1959) lists a large number of causes in the newborn but omits any reference to purpura fulminans. However, it is well recognized that thrombocytopenia may be associated with other deficiencies as in this infant. Platelet counts in the newborn do not differ much from those in older subjects, ranging from 150,000 to 350,000 per c.mm. A level below 100,000 per c.mm. is considered abnormally low. The platelet counts in this infant indicate significant thrombocytopenia.

It is probable that the deficiencies of both fibrinogen and platelets existed before birth because the baby was born with 'purpura'. The extent of the organization of the thrombi shown by microscopy also suggests that the process was one of some duration. Whatever parts may have been played by the family deficiency of fibrinogen and an unknown precipitating cause in the development of the disease, it probably started in utero.

In considering some common pathological mechanism which may deplete these coagulation factors, it is postulated that purpura fulminans is a disease of primary vascular damage with intravascular thrombosis. The relative severity of the depletion of coagulation factors is determined by the nature and rapidity of the thrombotic process, the rate of regeneration of fibrinogen, prothrombin and factor $\mathrm{V}$ by the liver and finally by the bone marrow for platelets. 
TABLE 2

VARIOUS BLOOD FINDINGS ON PARENTS AND SURVIVING CHILD

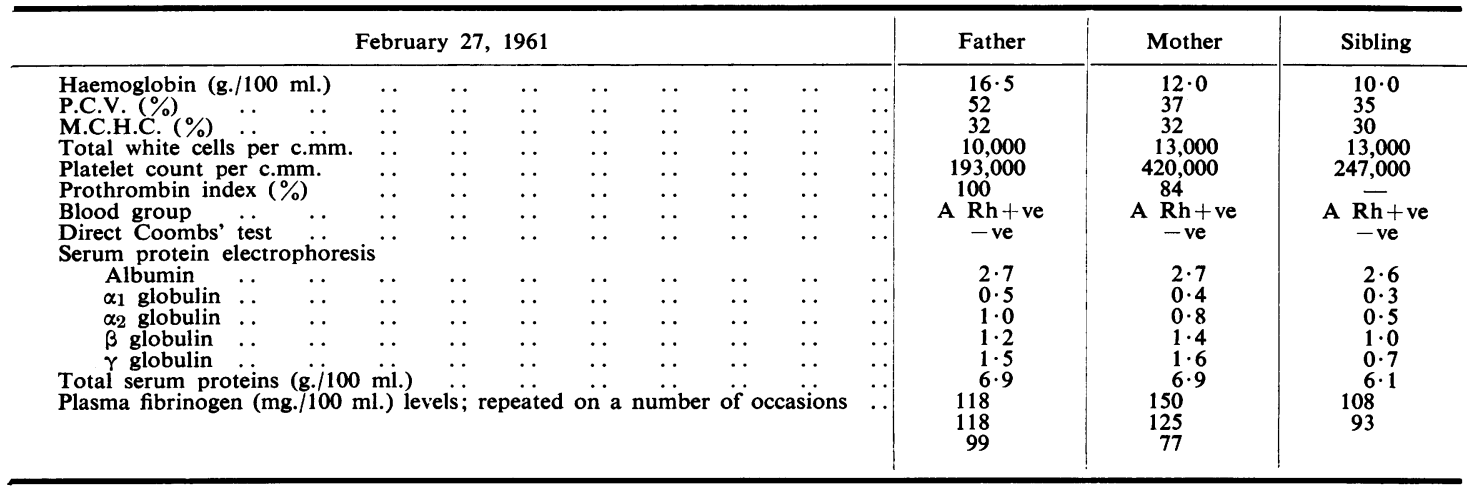

Relation of Purpura Fulminans to Other Antigen and Antibody Diseases. The syndromes of purpura fulminans, thrombotic thrombocytopenic purpura (thrombotic microangiopathic haemolytic anaemia or Moschcowitz's syndrome), periarteritis nodosa and anaphylactoid purpura (Henoch-Schonlein purpura) all have an obscure aetiology and pathogenesis. Many authors (Crawford and Riddler, 1959; Heal and Kent, 1953; Chambers et al., 1952; Turin et al., 1959; Seinfeld et al., 1960; Balf, 1951; Kingsley and Aquino, 1958; Craig and Gitlin, 1957; Wile and Sturgeon, 1956; Gairdner, 1948; Robinson, Orchard and Eden, 1958; Vernier, Worthen, Peterson, Colle and Good, 1961) feel that the primary fault appears to be a focal vascular lesion with swelling of the endothelium, rupture of the vessel wall and haemorrhage. These authorities have suggested independently that each disease is primarily a vascular disorder caused by an abnormal immunological mechanism of unknown origin. They have claimed a similarity to the Shwartzman phenomenon.

In the Shwartzman phenomenon, the repeated administration of endotoxin to experimental animals produces the tissue lesions of a hypersensitivity reaction. Although opinion is divided, it is probable that such a reaction can occur in man. Balf (1951) discusses the alimentary lesion in anaphylactoid purpura where a large part of the intestinal tract is affected. It is interesting to note also that many sections of the gastro-intestinal tract are similarly damaged in the generalized Shwartzman reaction. The immunological reaction that occurs in the Shwartzman phenomenon implies the presence of a provocative antigen such as a bacterium, usually from an exogenous source. However, modern concept centres on autoimmune disease mechanisms.
No attempt will be made to speculate what part autoimmune disease might have played in the production of the illness in the case reported. The history of 'purpura' at birth in three successive siblings (presumably having the same disease) raises the possibility that a maternal-foetal immunological mechanism may have played some role in the cause of the disease.

In an interesting experimental study, McKay, Kliman and Alexander (1959) have shown that during the Shwartzman reaction, blood fibrinogen decreases during the time when intravascular thrombi are deposited. Thomas (1961) states that in the generalized Shwartzman reaction, blood fibrinogen is depleted to such an extent as to constitute nearly complete afibrinogenaemia. It is conceivable that similar deficiencies in other coagulation factors occur when intravascular thrombi are deposited.

Diagnosis and Treatment. From the evidence available, it appears that there is a clinical paradox of haemorrhagic diathesis appearing simultaneously with intravascular thrombosis. The action of heparin in preventing the thrombosis results in the conclusion that in certain instances, such as purpura fulminans, a case presenting as a haemorrhagic disease should be treated with an anticoagulant. Furthermore, shock should be treated, and blood loss should be replaced by transfusion as also should deficient coagulation factors.

An accurate diagnosis is very difficult to make before treatment is instituted. It should be based on the appearance of the lesions and the knowledge that the condition of purpura fulminans exists. Differentiation must be made from other forms of gangrene. Once such a case has been seen it is unlikely to be mistaken for any other condition.

(1)


Adrenocortical preparations and antibiotics do not seem to be of help in therapy.

\section{Summary}

Clinical details are given of purpura fulminans in a newborn infant who died on the eighth day after birth.

As far as can be ascertained, this is the first reported case of purpura fulminans in a newborn infant.

Intravascular thrombosis and extensive extravascular haemorrhage was associated with thrombocytopenia and hypofibrinogenaemia. Gangrene and haemorrhage affected the skin as well as the viscera. There was evidence to suggest that the disease process started in utero.

The pathology of purpura fulminans and its resemblance to anaphylactoid purpura, periarteritis nodosa and thrombotic thrombocytopenic purpura are discussed. The four diseases seem to have a common relation to the hypersensitive immunological reaction which is found in the Shwartzman phenomenon.

I wish to express my thanks to Professor H. L. Wallace, Dr. F. Walt, Dr. N. M. Mann and Dr. J. C. Simson for constructive criticism and encouragement in the preparation of this paper; and to Professor J. Wainwright for the Pathological Report; also to Dr. S. Disler, Medical Superintendent, for facilities, and to Mr. C. R. Stuart for the photographs, excluding Figs. 2 and 3.

\section{REFERENCES}

Balf, C. L. (1951). The alimentary lesion in anaphylactoid purpura. Arch. Dis. Childh., 26, 20

Brühl, H. (1931). Beitrag zum Krankheitsbild der Purpura fulminans. Z. Kinderheilk., 50, 547. Cited by Heal and Kent (1953).

Chambers, W. N., Holyoke, J. B. and Wilson, R. F. (1952). Purpura fulminans. Report of two cases following scarlet fever. New Engl. J. Med., 247, 933.

Craig, J. M. and Gitlin, M. D. (1957). The nature of the hyaline thrombi in thrombotic thrombocytopenic purpura. Amer. $J$. Path., 33, 251 .

Crawford, S. E. and Riddler, J. G. (1959). Purpura fulminans. Amer. $J$. Dis. Child., 97, 198.

Dick, G. F., Miller, E.' M. and Edmondson, H. (1934). Severe purpura with gangrene of lower extremity following scarlet fever; recovery after amputation. ibid., 47, 374.
Dunn, H. G. (1951). Gangrenous purpura and its occurrence in meningococcal septicemia. Arch. Dis. Childh., 26, 184.

Dyggve, H. (1947). Case of purpura fulminans with fibrinogenopenia in association with scarlatina. Acta med. scand., 127, 382

Glueck, H. I. and Sutherland, J. M. (1961). Inherited Factor VII, defect in a negro family. Pediatrics, 27, 204.

Gairdner, D. (1948). The Schönlein-Henoch syndrome (anaphylactoid purpura), Quart. J. Med., 17, 95.

Heal, F. C. and Kent, G. (1953). Purpura fulminans with afibrino-

genaemia. Canad. med. Ass. J., 69, 367.
Hunt, L. W. (1938). Hemorrhagic purpura in scarlet fever. Amer. J. Dis. Child., 56, 1086.

Kaplan, E. (1959). Congenital and neonatal thrombocytopenic purpura. A review. J. Pediat., 54, 644.

Kingsley, J. W. and Aquino, R. B. (1958). Thrombotic thrombocytopenic purpura: report of a case with review of the literature. Ann. intern. Med., 49, 934.

Knauer, H. (1927). Die Krankheitsbild der Purpura fulminans. $J b$. Kinderheilk., 118, 1. Cited by Heal and Kent (1953).

Koller, F., Gasser, C., Krüsi, G. and de Muralt, G. (1950). Purpura fulminans nach Scharlach mit Faktor V-Mangel und Antithrominüberschuss. Acta haemat. (Basel), 4, 33.

Little, J. R. (1959). Purpura fulminans treated successfully with anticoagulation. J. Amer. med. Ass., 169, 36.

Marthaler, P., Pickel, R, and Bütler, R. (1957). Beitrag zur Kenntnis der Purpura fulminans. Schweiz. med. Wschr. 87, 657 .

McConnell, G. and Weaver, H. L. (1922). Purpura fulminans during convalescence from scarlet fever. J. Amer. med. Ass., $78,165$.

McKay, D. G., Kliman, A. and Alexander, B. (1959). Experimental production of afibrinogenemia and hemorrhagic phenomena by combined fibrinolysis and disseminated intravascular coagulation. New Engl. J. Med., 261, 1150 .

Poe, M. (1959). Clot observation test for clinical diagnosis of clotting defects. Anesthesiology, 20, 825.

Rausen, A. A. and Diamond, L. K. (1961). 'Enclosed' hemorrhage and neonatal jaundice. Amer. J. Dis. Child., 101, 164.

Robinson, M. J., Orchard, N. P. and Eden, A. R. (1958). Thrombotic microangiopathic hemolytic anaemia. Arch. Dis. Childh., 33,520 .

Schulman, I. and Smith, C. H. (1957). Coagulation disorders in infancy and childhood. Advanc. Pediat., 9, 231.

Seinfeld, R. H., Hennigar, R. and Hellman, L. M. (1960). Purpura fulminans complicating pregnancy. Amer. J. Obstet. Gynec., 80,161 .

Sheldon, J. H. (1947). Purpura necrotica. A possible clinical application of the Shwartzman phenomenon. Arch. Dis. Childh., 22, 7.

Stirland, R. M. (1956). A rapid method of estimating fibrinogen. Lancet, 1, 672 .

Stoesser, A. V. and Lockwood, W. W. (1938). Varicella complicated with acute thrombocytopenic purpura and gangrene. J. Pediat., 12,641 .

Taylor, K. B. and Wright, F. W. (1956). Purpura gangrenosa. Lancet, 2, 284.

Thomas, L. (1961). Role of endotoxins in immune and vascular reactions. Gastroenterology, 40, 364.

Turin, R. D., Mandel, S. and Hornstein, L. (1959). Fulminating purpura with gangrene of lower extremity necessitating amputation. J. Pediat., 54, 206.

van Creveld, $S$. (1959). Coagulation disorders in the newborn period. ibid., 54, 633.

Vernier, R. L., Worthen, H. G., Peterson, R. D., Colle, E. and Good, R. A. (1961). Anaphylactoid purpura. Pediatrics, 27, 181.

Webb, B. D., Dubbs, E. J. and Conrad, E. (1947). Postscarlatinal gangrene with prolonged prothrombin time. J. Pediat., 30, 76.

Wile, S. A. and Sturgeon, P. (1956). Thrombotic thrombocytopenic purpura; review of the subject with report of three cases in children. Pediatrics, 17, 882. 